\title{
DISTRIBUTED WIDEBAND SPECTRUM SENSING FOR COGNITIVE RADIO NETWORKS
}

\author{
Rocio Arroyo-Valles ${ }^{\star}$, Sina Maleki ${ }^{\dagger}$, and Geert Leus ${ }^{\star}$ \\ * Faculty of EEMCS, Delft University of Technology, The Netherlands \\ e-mail:\{m.d.r.arroyovalles,g.j.t.leus\}@tudelft.nl \\ ${ }^{\dagger}$ Interdisciplinary Centre for Security, Reliability and Trust, University of Luxembourg, Luxembourg \\ e-mail:sina.maleki@uni.lu
}

\begin{abstract}
Wideband spectrum sensing improves the agility of spectrum sensing and spectrum hand-off in cognitive radio systems. In this paper, a distributed wideband spectrum sensing technique over adaptive diffusion networks is proposed. Considering unknown and different channels between the primary and the cognitive users, an averaged received power spectrum across all the cognitive users is estimated by each user using diffusion adaptation techniques. This averaged power spectrum estimate is reliable enough for the users to perform spectrum sensing and make a decision regarding the presence or the absence of the primary user. The simulation results show that the detection performance of the system improves with the number of iterations. Further, a satisfactory detection performance at low SNRs is achieved after a few iterations, which is a desired characteristic for cognitive radio systems. Finally, it is shown that the cooperative technique outperforms the non-cooperative one in terms of estimation accuracy and detection performance.
\end{abstract}

Index Terms - Adaptive networks, distributed estimation, wideband spectrum sensing, cognitive radio.

\section{INTRODUCTION}

Reliable and agile spectrum sensing is a key functionality of a cognitive radio (CR) system [1]. Wideband spectrum sensing techniques improve the agility of the sensing process because cognitive users search among multiple channels at the same time. Further, in case a primary user (PU) returns to a currently empty band, spectrum hand-off becomes faster.

Recently, cooperative distributed detection schemes without a fusion center (FC) become more and more attractive [2][3][4]. Cooperative detection schemes are proposed in order to exploit the spatial diversity, thereby improving the detection performance of a CR network. Since CR networks generally consist of lowpower sensors, communication and energy constraints are critical limitations. Therefore, detection schemes where no FC is needed become appealing solutions. The focus of this paper is to propose a new wideband sensing technique which gives a reliable detection result in a short time, where no FC is necessary in scenarios where the channel between the primary user and the cognitive radios is unknown. Wideband spectrum sensing has been studied before in the literature. A wideband spectrum sensing technique based

The work was partially supported by projects PRI-PIBIN-2011-1266 and TEC2011-22480, the Spanish Ministry of Education, under the National Program of Human Resources Mobility from the I+D+i 2008-2011 National plan, the NWO-STW under the VICI program (project 10382), and the National Research Fund, Luxembourg under the project CO2SAT. on cooperative power spectrum estimation with sub-Nyquist rate samples is considered in [5]. In [6], a wideband spectrum sensing framework which identifies secondary transmission opportunities over multiple non-overlapping narrowband channels is proposed. The system is, however, complex because of the large number of required bandpass filters. A FC-based wideband power spectrum sensing technique is advocated in [7], where the FC is able to reconstruct the power spectrum from a few single bits with a good accuracy. The authors of [2] present a distributed spectrum sensing scheme based on the consensus of the CR users to make the final decision about the presence or the absence of the PU. The work in [8] addresses the problem of the cooperative estimation of the power distribution in space and frequency, which is carried out exploiting sparsity and through a basis expansion model of the power spectral density (PSD) map at arbitrary locations in space. In [9], the authors propose a distributed spectrum sensing algorithm over adaptive networks using diffusion adaptation. However, they assume that the channel and the signal are known for every user, and what is unknown is the presence or the absence of the signal. Also, the authors of [4] employ adaptive diffusion techniques to estimate the power spectrum in order to leverage a dynamic resource allocation process. The PSD of the primary user, which is represented as a linear combination of some preset basis functions, is estimated from the observed noisy measurements of the PSD via diffusion adaptation. However, the channel between the primary user and CR sensors is assumed to be known.

In this paper, distributed wideband spectrum sensing over adaptive diffusion networks is considered. Similar to [4], we focus on distributed wideband PSD estimators. However, we follow a different approach as channels are unknown and different for every user, which is mostly the case in CR networks. Each sensor computes an autocorrelation vector by collecting $N$ noisy samples of the received signal at each iteration. This vector is then used in order to locally compute a power spectrum estimate in a least mean squares (LMS) fashion. As the PSD estimate does not need to be extremely accurate but precise enough to identify the bands used by the PU, an averaged received PSD estimate across all the sensors is then computed at every user. Cognitive users receive the current local PSD estimates of the immediate neighbors and combine them following a specific combination rule. The rule, which is known to all the sensors, can be chosen according to the available information at the sensors, and it can range from a simple averaging operation to a more intelligent weighted combination [10]. In this way, weighting the received power spectrum of neighbors can help those users with bad channels to improve their detection capabilities. We further propose three decision statistics to be compared with a 
threshold, $\lambda$, in order to decide about the presence or the absence of the PU at each user and in each iteration.

Diffusion adaptation techniques are usually employed to estimate a common parameter of interest (typically the PSD of the primary user in a detection scenario). However, simulation results will show that this strategy can be satisfactorily employed in order to estimate an average PSD in unknown channel scenarios, what is deemed to be good enough to perform the detection task.

The remainder of the paper is organized as follows. Distributed power spectrum estimation over adaptive diffusion networks is proposed in Section II. In Section III, the proposed estimated power spectrum is used in order to detect the presence or the absence of the primary user. Simulation results are provided in Section IV, and we draw our conclusions in Section V.

\section{POWER SPECTRUM ESTIMATION BASED ON ADAPTIVE DIFFUSION}

A network of $K$ nodes (cognitive radio users) is considered. The set of nodes connected to the node $k$, including itself, is the neighborhood of the node $k$, which is denoted as $\mathcal{N}_{k}$. The primary user signal is assumed to be a wide-sense stationary wideband signal denoted by $s(n)$. Denoting $x_{k}(n)$ to be the observation at node $k$ and time $n$, the received signal is modeled as $x_{k}(n)=h_{k}(n) * s(n)+v_{k}(n)=\sum_{j=0}^{p-1} h_{k}(j) s(n-j)+$ $v_{k}(n)$. Here, $v_{k}(n)$ denotes the additive-white-Gaussian noise, which follows an i.i.d. distribution with zero mean and variance $\sigma_{v_{k}}^{2}$, and $h_{k}(n)$ is the channel between the primary user and the sensor $k$, which is modeled as a finite impulse response (FIR) filter of length $p$. At iteration $m$, each sensor collects $N$ noisy observations, so that the $N \times 1$ observation vector $\mathbf{x}_{k, m}$ is defined as $\mathbf{x}_{k, m}=\left[x_{k}(N m), x_{k}(N m+1), \ldots, x_{k}(N m+N-1)\right]^{T}$ for $m=0,1,2, \ldots$, where $(\cdot)^{T}$ denotes the transpose operation.

We denote by $r_{k}(l)$ the autocorrelation of the received signal $x_{k}(n)$ at lag $l, r_{k}(l)=\mathbb{E}\left\{x_{k}(n) x_{k}^{*}(n-l)\right\}$, where $(\cdot)^{*}$ denotes the complex conjugate operation. The power spectrum of $x_{k}(n)$ is the discrete-time Fourier transform (DTFT) of the autocorrelation function $r_{k}(l), P_{k}(\omega)=\sum_{l=-\infty}^{\infty} r_{k}(l) e^{-j \omega l}$, where $P_{k}(\omega)$ is real and non-negative. Then, from $\mathbf{x}_{k, m}$, each sensor can build an estimate $\hat{\mathbf{R}}_{k, m}$ of the $N \times N$ autocorrelation matrix $\mathbf{R}_{k, m}=\mathbb{E}\left\{\mathbf{x}_{k, m} \mathbf{x}_{k, m}^{H}\right\}$, where the elements of $\hat{\mathbf{R}}_{k, m}$ are computed as $\left[\hat{\mathbf{R}}_{k, m}\right]_{i j}=\hat{r}_{k, m}(i-j)=\hat{r}_{k, m}^{*}(j-i)=\frac{1}{N} \sum_{\tau=0}^{N-l-1} \mathbf{x}_{k, m}(\tau+$ $l) \mathbf{x}_{k, m}^{H}(\tau)$ for $l \geq 0$.

As the columns of $\hat{\mathbf{R}}_{k, m}$ contain the same information (i.e., the $N$-lag autocorrelation), that information can be collected into the $(2 N-1) \times 1$ autocorrelation vector $\hat{\mathbf{r}}_{k, m}=$ $\left[\hat{r}_{k, m}(1-N) \ldots, \hat{r}_{k, m}(-1), \hat{r}_{k, m}(0), \hat{r}_{k, m}(1), \ldots, \hat{r}_{k, m}(N-1)\right]^{T}$. Since the autocorrelation vectors have finite size, only a windowed estimate of the power spectrum can be obtained as $\hat{P}_{k, m}(\omega)=\sum_{l=1-N}^{N-1} \hat{r}_{k, m}(l) e^{-j \omega l}$. Hence, if the frequency axis is discretized, a $(2 N-1)$-point estimate of the power spectrum can be obtained based on the following linear relationship

$$
\hat{\mathbf{p}}_{k, m}=\mathbf{F} \hat{\mathbf{r}}_{k, m},
$$

with $\hat{p}_{k, m}(f)=\hat{P}_{k, m}\left(\frac{2 \pi f}{2 N-1}\right)$, for $f=0,1, \ldots 2 N-1$ and $\mathbf{F}$ is the $(2 N-1) \times(2 N-1)$ discrete Fourier transform (DFT) matrix
Note that (1) can be rewritten as $\hat{\mathbf{r}}_{k, m}=\mathbf{F}^{H} \hat{\mathbf{p}}_{k, m}$, where $(\cdot)^{H}$ denotes the Hermitian operation.

Since the autocorrelation estimate depends on the observed data $\mathbf{x}_{k, m}, \hat{\mathbf{r}}_{k, m}$ is different for each iteration $m$, and so $\hat{\mathbf{p}}_{k, m}$. In addition, given that sensors have unknown different channels, it is hard to compute the PSD of the PU; however, each sensor can adaptively compute an averaged estimate of the received PSD using a diffusion adaptation strategy. Several diffusion adaptation schemes have been proposed in the literature (see [10] and references therein), and one of them is the adapt-then-combine (ATC) diffusion algorithm [11]. It consists of two steps. The first one involves local adaptation, where each sensor $k$ updates its local estimate $\boldsymbol{\varphi}_{k, m}$ using the combined estimate from the previous iteration, $\hat{\mathbf{p}}_{k, m-1}$, and its own data, $\hat{\mathbf{r}}_{k, m}$, as follows

$$
\boldsymbol{\varphi}_{k, m}=\hat{\mathbf{p}}_{k, m-1}+\mu_{k} \mathbf{F}\left[\hat{\mathbf{r}}_{k, m}-\mathbf{F}^{H} \hat{\mathbf{p}}_{k, m-1}\right]
$$

where $\mu_{k}$ is a constant positive step size, which is chosen to be sufficiently small to ensure convergence [10].

The second step is a combination stage where the intermediate estimates of the received power spectrum $\left\{\boldsymbol{\varphi}_{z, m}\right\}$ from the neighborhood of sensor $k\left(z \in \mathcal{N}_{k}\right)$ are combined through the coefficients $\left\{a_{z, k}\right\}$ to obtain the updated estimate $\hat{\mathbf{p}}_{k, m}$ as follows

$$
\hat{\mathbf{p}}_{k, m}=\sum_{z \in \mathcal{N}_{k}} a_{z, k} \boldsymbol{\varphi}_{z, m},
$$

where the combining weights $\left\{a_{z, k}\right\}$, collected into the $N \times N$ matrix $\mathbf{A}$ as $[\mathbf{A}]_{z, k}=a_{z, k}$, should satisfy $\mathbf{A 1}=\mathbf{1}$ and $a_{z, k}=0$ if $z \notin \mathcal{N}_{k}$, where $\mathbf{1}$ stands for a column vector with all elements equal to one. Note that different channel gains result in different received PSD at users, so that an averaged received PSD estimate across all the sensors is obtained in (3). Besides, as sensors only exchange estimates with their one-hop neighbors, the estimate $\hat{\mathbf{p}}_{k, m}$ is not exactly same (as in consensus techniques), but similar for all sensors $k$.

Several approaches have been proposed in the literature in order to select the combining weights. More specifically, denoting $\xi_{z, k, m}$ as the power spectrum estimation error (with regard to the true averaged PSD) of node $z$ available at node $k$, at iteration $m$, in this paper, following the idea of [10], we use a combination rule where node $k$ combines the local received power spectrum estimates from its neighbors in an inversely proportional manner to the estimation error

$$
a_{z, k, m}=\left\{\begin{array}{l}
\frac{\xi_{z, k, m}^{-2}}{\sum_{j \in \mathcal{N}_{k}} \xi_{j, k, m}^{-2}} \text { if } z \in \mathcal{N}_{k} \\
0 \text { otherwise }
\end{array}\right.
$$

As the power spectrum estimation errors from the neighbors of node $k$ are unavailable (and change every iteration), node $k$ can estimate them recursively from its own previous estimate as

$$
\hat{\xi}_{z, k, m}^{2}=\left(1-\eta_{k}\right) \hat{\xi}_{z, k, m-1}^{2}+\eta_{k}\left\|\boldsymbol{\varphi}_{z, m}-\hat{\mathbf{p}}_{k, m-1}\right\|_{2}^{2},
$$

where $\eta_{k}$ is a small positive step size. In this combination rule, nodes with smaller estimation errors will be given larger weights.

\section{SPECTRUM SENSING USING POWER SPECTRUM ESTIMATION}

In this section, we present the detection algorithm which is employed by the cognitive users to determine the presence or the absence of the primary user. Denoting $\mathcal{H}_{1}$ and $\mathcal{H}_{0}$ as the hypothesis 
representing the presence and the absence of the primary user, respectively, our goal is to solve the following binary hypothesis testing problem

$$
\begin{aligned}
& \mathcal{H}_{0}: x_{k}(n)=v_{k}(n) \quad k=1,2, \ldots K \\
& \mathcal{H}_{1}: x_{k}(n)=h_{k}(n) * s(n)+v_{k}(n) \quad k=1,2, \ldots K .
\end{aligned}
$$

The channel is generally not known at the cognitive radios. Therefore, the cognitive sensors need to use detection techniques which perform well when the channel state information is not available or not perfect. To employ the earlier discussed power spectrum estimation technique for spectrum sensing is then a good strategy in order to tackle the lack of channel state information at the cognitive sensors. Here, we employ the estimate in (3) in order to solve the binary hypothesis problem in (6). Denoting $\mathcal{S}$ as the set of available estimated frequencies in the band of interest, three possible decision statistics can be: (a) $\mathcal{T}_{k, m}=\frac{1}{|S|} \sum_{f_{s} \in \mathcal{S}} \hat{p}_{k, m}\left(f_{s}\right)$, (b) $\mathcal{T}_{k, m}=\max _{f_{s} \in \mathcal{S}} \hat{p}_{k, m}\left(f_{s}\right)$, and (c) $\mathcal{T}_{k, m}=\hat{p}_{k, m}\left(f_{c}\right)$, where $\mathcal{T}_{k, m}$ is the decision statistic, and $f_{c}$ is the carrier frequency of the desired band. This way, the decision regarding the presence or the absence of the primary user at each sensor is made locally by the following rule $\mathcal{T}_{k, m} \underset{\mathcal{H}_{0}}{\stackrel{\mathcal{H}_{1}}{\gtrless}} \lambda_{k, m}$, with $\lambda_{k, m}$ denoting the detection threshold at sensor $k$ and iteration $m$. Note that although the decision about the presence of the PU is made locally by each sensor, it becomes the global one due to the fact that sensors converge towards the desired estimate with acceptable mean square error bounds using the diffusion technique. The associated probabilities of false alarm and detection, denoted by $P_{f, k, m}$ and $P_{d, k, m}$, respectively, are then defined as $P_{f, k, m}=\operatorname{Pr}\left(\mathcal{H}_{1} \mid \mathcal{H}_{0}\right)=\operatorname{Pr}\left(\mathcal{T}_{k, m} \geq \lambda_{k, m} \mid \mathcal{H}_{0}\right)$ and $P_{d, k, m}=\operatorname{Pr}\left(\mathcal{H}_{1} \mid \mathcal{H}_{1}\right)=\operatorname{Pr}\left(\mathcal{T}_{k, m} \geq \lambda_{k, m} \mid \mathcal{H}_{1}\right)$.

\section{SIMULATIONS}

In this section, we evaluate the performance of the proposed scheme. Two approaches have been compared; the non-cooperative one (sensors do not exchange information with their neighbors and estimate the received PSD at each sensor, i.e., $\hat{\mathbf{p}}_{k, m}=\boldsymbol{\varphi}_{k, m}$ ) and the cooperative one, where the parameter $\eta_{k}$ in (5) is set to 0.2 and $\mu_{k}=0.1$ for all the sensors. The cognitive network consists of $K=15$ nodes with a topology as shown in Fig. 1 .

We consider a complex baseband representation of an OFDM signal, with a wide frequency band of interest, ranging from -40 to $40 \mathrm{MHz}$, which contains 10 non-overlapping channels of equal bandwidth $(8 \mathrm{MHz})$. Each symbol has 64 frequencies but only 52 are activated, with 16-QAM modulation and a cyclic prefix length of 16 . The oversampling factor is 16 . Some of the 10 nonoverlapping channels might not be used by primary users and hence, are available for secondary transmissions.

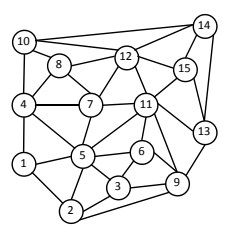

Fig. 1. Network topology

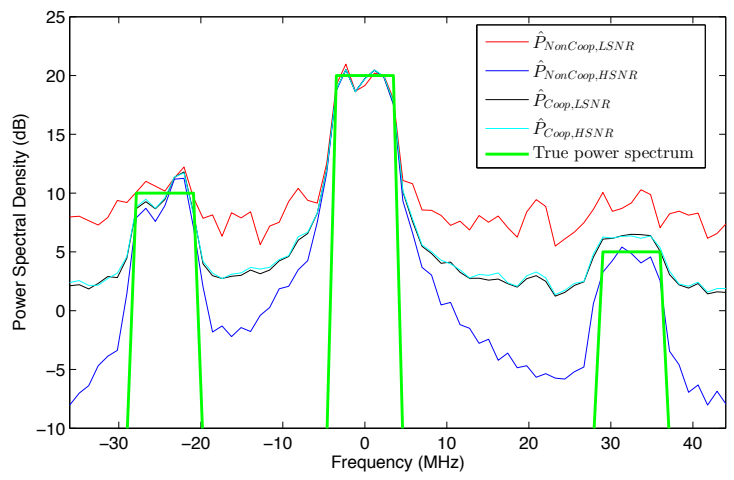

Fig. 2. True power spectrum $\left(\mathbf{p}_{s}\right)$ and the estimated power spectrum for the cooperative and non-cooperative schemes for those sensors having the highest and the lowest SNR (HSNR and LSNR).

\section{IV-A. The averaged estimated power spectrum density}

Initially, we analyze how good the averaged received PSD estimate is regarding the true PSD to verify if each user should rely on the obtained estimate to perform detection. Note that our main goal is not designing a good PSD estimator but performing detection satisfactorily by first identifying the occupied bands. The signal $s(n)$ is corrupted by additive white Gaussian noise $v_{k}(n)$, whose variance $\sigma_{v_{k}}^{2}$ varies from one sensor to another so that the SNR at each node is taken from a uniform distribution between $[0,20] \mathrm{dB}$. We consider two types of channels $h_{k}(n)$. The first case is a static one-tap FIR filter whose gain is constant $\left(h_{k}=1\right)$ during all time instants. The second case is a block-fading timevarying one-tap FIR filter, modeled as an i.i.d. zero mean random Gaussian variable with variance $\sigma_{h}^{2}=1$. Here, only three channels are occupied with different power levels $(10 \mathrm{~dB}, 20 \mathrm{~dB}$ and $5 \mathrm{~dB})$.

The metric which is used to compare the quality of the estimate is the network normalized mean square deviation (NMSD), which is defined as

$$
N M S D=\frac{1}{K} \sum_{k=1}^{K} \frac{\left\|\hat{\mathbf{p}}_{k}-\mathbf{p}_{s}\right\|^{2}}{\left\|\mathbf{p}_{s}\right\|^{2}}
$$

where $\mathbf{p}_{s}$ is the true power spectrum density (corresponding to the non-noisy signal $s(n)$ ).

Fig. 2 depicts the true power spectrum and the averaged received power spectrum obtained by the cooperative and non-cooperative approaches where the number of collected samples at each iteration is $N=64$ considering a static channel. The spectra corresponding to the sensor with the highest and lowest SNR (19.65 dB and $0.31 \mathrm{~dB}$, respectively) have been plotted. The presence of active frequency bands is well-estimated and the quality of the estimation is satisfactory. We can see that employing the diffusion algorithm, the power spectrum estimation at nodes with different SNRs reaches almost the same value after a number of iterations. This is clearly not observed for the non-cooperative network.

Fig. 3 shows the average evolution of the network NMSD for 1000 realizations when $\sigma_{v_{k}}^{2}$ varies, so that the SNR at each node is taken from a uniform distribution between $[-10,10] \mathrm{dB}$, for both the static and the block-fading time-varying channel. It can be observed that the estimation error achieved by the cooperative scheme is lower than the error achieved by the non-cooperative 


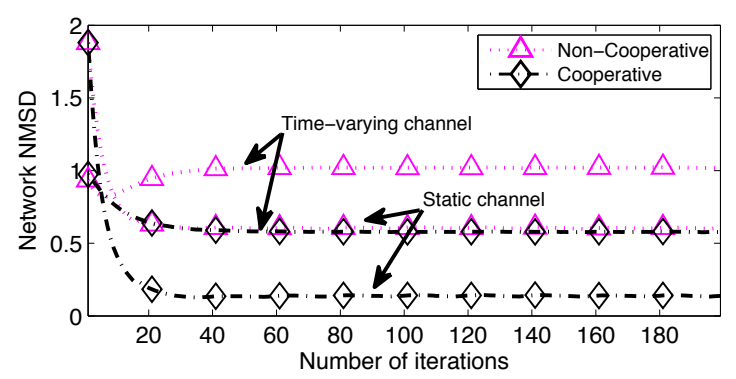

Fig. 3. Learning curve of the network NMSD for a static and a blockfading time-varying channel.

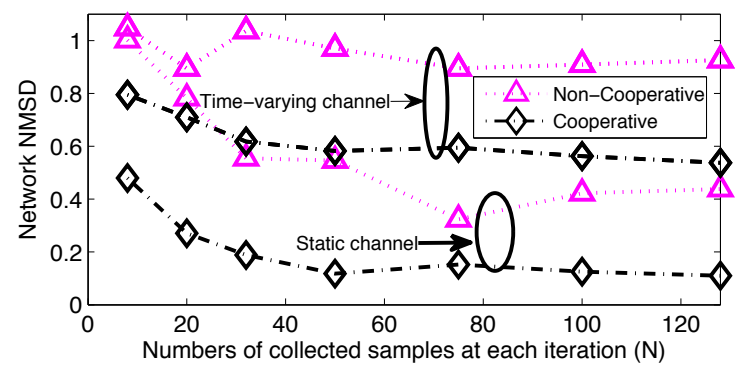

Fig. 4. Network NMSD for different number of collected observations at each iteration $(\mathrm{N})$ and channel types.

technique. As expected, the estimation error is higher when a timevarying channel is considered.

Besides, the network NMSD (for steady state), which is averaged over 1000 Monte-Carlo simulations, has been analyzed for different numbers of collected samples at each iteration and is illustrated in Fig. 4 for both the static and the block-fading time-varying channel. According to the previous results, the network NMSD achieved by the cooperative approach is lower than the non-cooperative one. Further, and as can be expected, the higher the $N$ value is, the lower the estimation error is, because more samples are used to compute the autocorrelation.

\section{IV-B. Detection performance analysis}

The same CR network used for the estimation section is considered for the detection performance analysis (Fig. 1). The channel is assumed static and the static gain $h_{k}$ is computed so that the SNR of each sensor is randomly chosen between $-20 \mathrm{~dB}$ and $0 \mathrm{~dB}$, considering $\sigma_{v_{k}}^{2}=1$. The results are averaged over 1000 simulations where only one frequency band is activated. In order to detect the primary user, each sensor uses the decision statistic defined in (a), i.e., $\mathcal{T}_{k, m}=\frac{1}{|S|} \sum_{f_{s} \in \mathcal{S}} \hat{p}_{k, m}\left(f_{s}\right)$. Further, the hypothesis testing problem is applied as data arrive at each iteration.

The probability of detection and false alarm versus the number of iterations are depicted in Fig. 5, for the sensors with the highest and the lowest SNR. The detection threshold, which is set experimentally, and the number of collected observations at each iteration are set to $\lambda=0.19 \mathrm{~dB}$ and $N=64$, respectively, for all the sensors and iterations. It is shown that the probability of detection improves significantly as the number of iterations increases, while the probability of false alarm remains at an acceptable level (approximately 0 except for the sensor with the

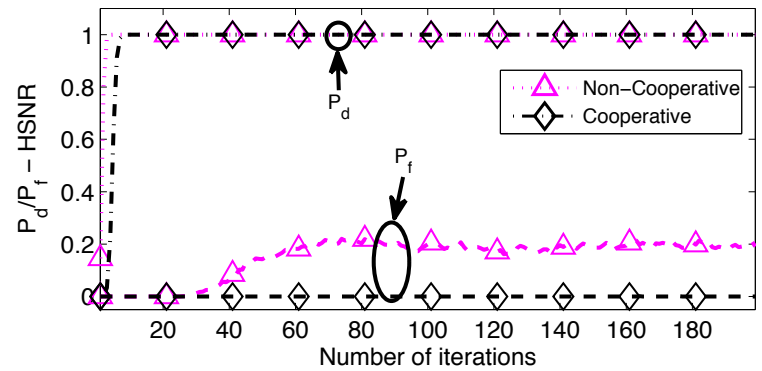

(a)

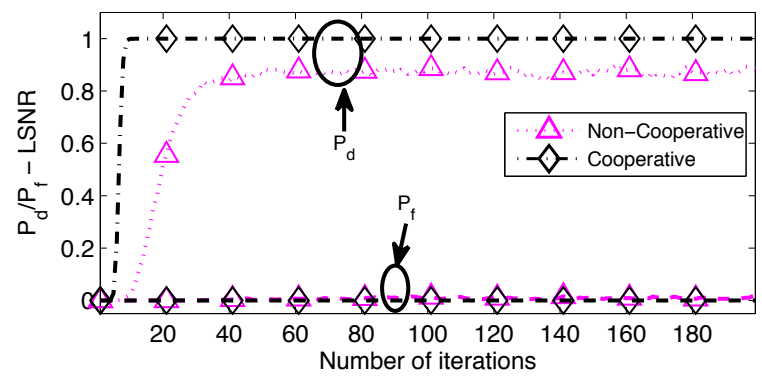

(b)

Fig. 5. Detection performance for the sensor with (a) the highest SNR, and (b) the lowest SNR, for SNR $=[-20,0] \mathrm{dB}$ and $\lambda=0.19 \mathrm{~dB}$.

highest SNR, which is around 0.2). Furthermore, it is observed that the adaptive cooperative spectrum sensing scheme outperforms the non-cooperative one, both in the probability of false alarm and the probability of detection. This way, we can see that distributed wideband spectrum sensing delivers a reliable sensing performance, while achieving a higher throughput than the single-user noncooperative scenario. Note that the probability of detection and false alarm in the cooperative scheme is similar for both sensors. The reason is that the local estimate at each sensor becomes the global estimate. Further, the convergence of the cooperative scenario is shown to be faster than the one for the non-cooperative scenario, except for the convergence rate of the probability of detection for the sensor with the highest SNR.

\section{CONCLUSIONS}

Distributed wideband spectrum sensing over adaptive diffusion networks in scenarios with unknown channels was explored in this paper. Every sensor computes a local received power spectrum estimate using a distributed LMS algorithm, which is later combined with those estimates obtained from their one-hop neighbors in order to obtain an averaged received power spectrum estimate among all the sensors. Later, a decision about the presence or the absence of the licensed user is made based on this power spectrum estimate. The simulation results showed that the proposed scheme has a good performance, in terms of both estimation and detection, outperforming the case when no cooperation among sensors is carried out. Future lines include to compare our method with other related state-of-the-art works, the systematic selection of the detection threshold, and the design of censoring strategies for not receiving estimates from those users with bad channels. 


\section{REFERENCES}

[1] E. Axell, G. Leus, E. G. Larsson, and H. V. Poor, "Spectrum sensing for cognitive radio: State-of-the-art and recent advances," IEEE Signal Processing Magazine, vol. 29, no. 3, pp. 101-116, 2012.

[2] Z. Li, F.R. Yu, and M. Huang, "A distributed consensus-based cooperative spectrum-sensing scheme in cognitive radios," IEEE Trans. on Vehicular Technology, vol. 59, no. 1, pp. 383393, 2010.

[3] K. Seung-Jun, E. Dall'Anese, and G.B. Giannakis, "Cooperative spectrum sensing for cognitive radios using kriged kalman filtering," IEEE Journal of Selected Topics in Signal Processing, vol. 5, no. 1, pp. 24-36, 2011.

[4] P. Di Lorenzo, S. Barbarossa, and A.H. Sayed, "Bio-inspired decentralized radio access based on swarming mechanisms over adaptive networks," IEEE Trans. on Signal Processing, vol. 61, no. 12, pp. 3183-3197, 2013.

[5] D.D. Ariananda, and G. Leus, "Cooperative compressive wideband power spectrum sensing," in Proc. of the Forty Sixth Asilomar Conference on Signals, Systems and Computers (ASILOMAR'12), 2012, pp. 303-307.

[6] P. Paysarvi-Hoseini, and N.C. Beaulieu, "Optimal wideband spectrum sensing framework for cognitive radio systems," IEEE Trans. on Signal Processing, vol. 59, no. 3, pp. 11701182, 2011.

[7] O. Mehanna, and N.D. Sidiropoulos, "Frugal sensing: Wideband power spectrum sensing from few bits," IEEE Transactions on Signal Processing, vol. 61, no. 10, pp. 2693-2703, 2013.

[8] J.A. Bazerque, and G.B. Giannakis, "Distributed spectrum sensing for cognitive radio networks by exploiting sparsity," IEEE Trans. on Signal Processing, vol. 58, no. 3, pp. 1847$1862,2010$.

[9] F.S. Cattivelli, and A.H. Sayed, "Distributed detection over adaptive networks using diffusion adaptation," IEEE Trans. on Signal Processing, vol. 59, no. 5, pp. 1917-1932, 2011.

[10] A.H. Sayed, Diffusion Adaptation over Networks. In EReference Signal Processing, R. Chellapa and S. Theodoridis, editors, Elsevier, 2013. Also available as arXiv:1205.4220v1, May 2012.

[11] F.S. Cattivelli, and A.H. Sayed, "Diffusion LMS Strategies for Distributed Estimation," IEEE Trans. on Signal Processing, vol. 58, no. 3, pp. 1035 -1048, march 2010. 